\title{
Effect of Anti Darkening Treatments and Packing Materials on Whole and Sliced Fruits of Frozen Aonla Processed Product cv. Gujarat Aonla-1
}

\author{
V.K. Patel ${ }^{*}$, B.H. Panchal and R.A. Khimani \\ Department of Horticulture, B.A. College of Agriculture, A.A.U, Anand, Gujarat, India \\ *Corresponding author
}

\section{A B S T R A C T}

\section{Key words \\ Frozen aonla, Candy and Juice, Different \\ packaging, Anti- darkening treatment \\ Article Info \\ Accepted: \\ 07 October 2018 \\ Available Online: \\ 10 November 2018}

\begin{abstract}
Aonla fruits have high nutritive values. Storage of fresh fruits as well as its products is essential to capture the emerging consumer market. With the aim of extending the postharvest life and preparation different product (Candy and Juice) by minimizing the loss of vitamin- $\mathrm{C}$ and optimizing organoleptic score, an experiment was conducted. Vitamin $\mathrm{C}$ content of aonla candy and juice were highest in whole fruits packed in aluminium foil bag after 4 and 8 months of storage. In organoleptic score (test and marketability) of candy and juice prepared after 4 and 8 months of storage, whole fruits, fruits treated with $1 \%$ ascorbic acid or $1 \%$ citric acid and aluminium foil bag packed frozen fruit's products (candy and juice) recorded maximum organoleptic score. Among the treatment combinations, whole fruit treated with $1 \%$ ascorbic acid and packed in aluminium foil bag recorded highest organoleptic score (test and marketability).
\end{abstract}

\section{Introduction}

Aonla or Indian gooseberry (Emblica officinalis Gaertn), a versatile tree of arid and semi-arid region, is adaptable in diverse soil and climatic conditions. Its fruits have high nutritive and therapeutic values. Importance of this tree is such that, it has been worshiped, and planting of tree around house is a sign of spirituality. Aonla is used in more than 50 products and ayurvedic formulations. Among these, aonla pulp, juice, squash, RTS beverage, jam, candy, powder etc. are more popular. Diversification of aonla products to suit the requirement of consumer is essential to capture the emerging market throughout the year. But fresh aonla can available around 3 to 4 months in market and it is perishable in nature. Its shelf life is around 6 to 8 days at room temperature.

The information on post-harvest management, packing and its different product is not sufficient. Quick freezing is one of the easiest, most convenient and least time-consuming ways for storage of fresh fruits. Freezing does not sterilize the fruits but extreme low temperature simply retards growth of microorganisms and slows down changes that affect quality. Properly frozen fruits will retain much of their fresh flavor and nutritive value. Their texture, however, may be somewhat soften than that of fresh fruit. This fruits are not suitable for fresh marketing but it is suitable for processing. Aonla fruits, will darken if their cut surfaces are exposed to air. 
Ascorbic acid and citric acid may be added to packing liquids or sprinkled on cut fruit surfaces to prevent darkening.

Therefore, an investigation was taken to study anti darkening treatments and packing material on quality of process product making from whole and slice frozen aonla fruits.

\section{Materials and Methods}

The present investigation was carried out at Department of Horticulture, B.A. College of Agriculture, Anand Agricultural University, Anand. The aonla variety 'Gujarat Aonla-1' was selected for the present study. The fruits were selected from a single tree for uniformity in fruits. The treatments include preservative treatments, $\mathrm{C}_{1}: 1 \%$ ascorbic acid, $\mathrm{C}_{2}: 1 \%$ citric acid, $\mathrm{C}_{3}$ : control (water) preparation types $\left(\mathrm{F}_{1}\right.$ : whole fruit, $\mathrm{F}_{2}$ : sliced fruit), preservative treatments, $\mathrm{C}_{1}: 1 \%$ ascorbic acid, $\mathrm{C}_{2}: 1 \%$ citric acid, $\mathrm{C}_{3}$ : control (water) and three packing materials $\left(\mathrm{P}_{1}\right.$ : aluminum foil bag, $\mathrm{P}_{2}: 200$ gauge polyethylene bag, $\mathrm{P}_{3}$ : inner side laminated paper box). The treated fruits were quick frozen at $-30^{\circ} \mathrm{C}$ for 12 hours and stored at $-18^{\circ} \mathrm{C}$ in cold storage in two lots. First lot was taken out from cold storage after four months $\left(T_{2}\right)$ while the second lot was taken out after eight months $\left(\mathrm{T}_{3}\right)$. After processing (preparation of Candy and Juice) analyze the ascorbic acid (mg/100 g) and their organoleptic score for taste and marketability.

Ascorbic acid was determined as per standard procedures of Rangana (1979). Candy was made as per method described by Rai and Kikani (2003) and Juice was prepared using method described by Singh and Sanjeev (1995). Taste and marketability were assessed by panel of scientists. After compilation of test marks was given as per ranking, for Very $\operatorname{good}=10$, Good $=7.5$, Normal $=5$ and for Not good $=2.5$. The trial was laid out in a Complete Randomized Block Design with factorial concept. The data were subjected to statistical analysis.

\section{Results and Discussion}

\section{Vitamin-C content of candy (mg/100 g)}

The individual effect of treatments $\mathrm{F}, \mathrm{P}$ and $\mathrm{T}$ gave significant result (Table 1). Candy prepared from $F_{1}$ fruit, packed in $P_{1}$ (Aluminum foil bag) and prepared from fresh fruit $\left(\mathrm{T}_{1}\right)$ had significantly more vitamin $\mathrm{C}$ content $(170.02,170.00$ and $203.07 \mathrm{mg} / 100 \mathrm{~g})$ respectively.

The results of interaction effects of $\mathrm{F} \times \mathrm{P}, \mathrm{F} \times \mathrm{T}$ (Table 2) and PxT (Table 3) on vitamin $\mathrm{C}$ content of candy were significant. In $\mathrm{F} \times \mathrm{P}$ interaction, the $\mathrm{F}_{1} \mathrm{P}_{1}$ combination gave highest (174.59 mg/100g) vitamin C content of candy. This may be because of $F_{1}$ fruit in $\mathrm{P}_{1}$ packing maintained more vitamin $\mathrm{C}$ content due to less oxidation and respiration and therefore, candy prepared from this fruit contained more vitamin $\mathrm{C}$ during processing. In $\mathrm{F} \times \mathrm{T}$ interaction, treatment combination $\mathrm{F}_{1} \mathrm{~T}_{1}$ had higher $(203.26 \mathrm{mg} / 100 \mathrm{~g})$ vitamin $\mathrm{C}$ content of candy, which was at par with $\mathrm{F}_{2} \mathrm{~T}_{1}$. In $\mathrm{P} \times \mathrm{T}$ interaction the maximum vitamin $\mathrm{C}$ content of candy $(203.86 \mathrm{mg} / 100 \mathrm{~g})$ was observed in treatment combination of $\mathrm{P}_{3} \mathrm{~T}_{1}$, which was at par with $\mathrm{P}_{1} \mathrm{~T}_{1}$ and $\mathrm{P}_{2} \mathrm{~T}_{1}$. This may be due to at storage time $T_{1}$, packed fruit was fresh. After 4 and 8 month of storage, the highest vitamin $\mathrm{C}$ content of candy was found with treatment combinations of $\mathrm{P}_{1} \mathrm{~T}_{2}$ and $\mathrm{P}_{1} \mathrm{~T}_{3}$. (168.24 and $138.37 \mathrm{mg} / 100 \mathrm{~g}$ ) respectively. This may be due to the fact that packing $\mathrm{P}_{1}$ maintained more vitamin $\mathrm{C}$ content by controlling the physiological processes like oxidation and respiration. From storage durations, fresh fruits candy recorded significantly higher vitamin $\mathrm{C}$ than candy prepared from fruits stored for four and eight months. Reduction in vitamin $\mathrm{C}$ content during storage periods could be attributed to the conversion of $\mathrm{L}$ - 
ascorbic acid into dihydro-ascorbic acid in the presence of ascorbinase enzyme (Mapson, 1970). These results are in agreement with findings of Singh et al., (1987), Nath et al., (1992) and Patra and Sadhu (1992).

The interaction effect of $\mathrm{F} \times \mathrm{C} \times \mathrm{T}$ (Table 4) gave significant result. Maximum vitamin $\mathrm{C}$ content of candy observed in $\mathrm{F}_{1} \mathrm{C}_{1} \mathrm{~T}_{1}(203.41$ $\mathrm{mg} / 100 \mathrm{~g}$ ) which was at par with $\mathrm{F}_{1} \mathrm{C}_{2} \mathrm{~T}_{1}$, $\mathrm{F}_{1} \mathrm{C}_{3} \mathrm{~T}_{1}, \quad \mathrm{~F}_{2} \mathrm{C}_{1} \mathrm{~T}_{1}, \quad \mathrm{~F}_{2} \mathrm{C}_{2} \mathrm{~T}_{1} \quad$ and $\quad \mathrm{F}_{2} \mathrm{C}_{3} \mathrm{~T}_{1}$ treatments. After 4 month of storage, vitamin $\mathrm{C}$ content was maximum $(168.05 \mathrm{mg} / 100 \mathrm{~g})$ in $\mathrm{F}_{1} \mathrm{C}_{2} \mathrm{~T}_{2}$ and it was at par with $\mathrm{F}_{1} \mathrm{C}_{1} \mathrm{~T}_{2}$ and $\mathrm{F}_{1} \mathrm{C}_{3} \mathrm{~T}_{2}$. After 8 month of storage, treatment combination of $\mathrm{F}_{1} \mathrm{C}_{1} \mathrm{~T}_{3}$ gave maximum $(140.78 \mathrm{mg} / 100 \mathrm{~g})$ vitamin $\mathrm{C}$ content of candy which was at par with $\mathrm{F}_{1} \mathrm{C}_{2} \mathrm{~T}_{3}$ and $\mathrm{F}_{1} \mathrm{C}_{3} \mathrm{~T}_{3}$.

This may be due to the fact that after $4\left(\mathrm{~T}_{2}\right)$ and $8 \quad\left(\mathrm{~T}_{3}\right)$ months of storage, fruit $\mathrm{F}_{1}$ contained more vitamin $\mathrm{C}$ due to slower oxidation and respiration rate irrespective of chemicals (Thakur and Arya, 1988) and therefore candy prepared from those fruit maintain more vitamin $\mathrm{C}$.

\section{Vitamin-C content of juice (mg/100 g)}

The individual effect of treatments $\mathrm{F}, \mathrm{P}$ and $\mathrm{T}$ (Table 5) gave significant result. Juice prepared from $F_{1}$ (Whole fruits) fruit, packed in $\mathrm{P}_{1}$ (Aluminum foil bag) and prepared at time $\mathrm{T}_{1}$ (from fresh fruit) had significantly more vitamin $\mathrm{C}$ content $(546.58,539.30$ and $603.91 \mathrm{mg} / 100 \mathrm{~g}$ ) respectively.

The $\mathrm{F} \times \mathrm{T}$ and $\mathrm{P} \times \mathrm{T}$ (Table 6) interactions, vitamin $\mathrm{C}$ content of aonla juice gave significant result. The $\mathrm{F} \times \mathrm{P}$ interaction showed that, treatment combination of $\mathrm{F}_{1} \mathrm{P}_{1}$ contained significantly the highest $(558.38 \mathrm{mg} / 100 \mathrm{~g})$ vitamin $C$ content of juice $I n P \times T$ interaction treatment combinations, $\mathrm{P}_{1} \mathrm{~T}_{1}$ had significantly higher $(618.38 \mathrm{mg} / 100 \mathrm{~g})$ vitamin $\mathrm{C}$ content of juice. Here also with increased in storage period from $T_{1}$ to $T_{3}$, there was significant reduction in vitamin $\mathrm{C}$ content of juice in all the three packing treatments. However, fruit stored in $\mathrm{P}_{1}$ showed significantly less reduction in vitamin $\mathrm{C}$ content of juice as compared to $\mathrm{P}_{2}$ and $\mathrm{P}_{3}$. This may be due to fruit packed in $\mathrm{P}_{1}$ had less oxidation and respiration rate during the storage period.

The $\mathrm{F} \times \mathrm{C} \times \mathrm{T}$ and $\mathrm{F} \times \mathrm{P} \times \mathrm{T}$ (Table 7) interactions exhibit significant variation for vitamin $\mathrm{C}$ content of juice of fresh and frozen aonla during the experiment. In $\mathrm{F} \times \mathrm{C} \times \mathrm{T}$ interaction, treatment combination of $\mathrm{F}_{1} \mathrm{C}_{1} \mathrm{~T}_{1}$ gave maximum $(629.56 \mathrm{mg} / 100 \mathrm{~g})$ vitamin $\mathrm{C}$ content of juice, which was at par with $\mathrm{F}_{1} \mathrm{C}_{2} \mathrm{~T}_{1}$ and $\mathrm{F}_{1} \mathrm{C}_{3} \mathrm{~T}_{1}$. In $\mathrm{F} \times \mathrm{P} \times \mathrm{T}$ interaction, treatment combination of $\mathrm{F}_{1} \mathrm{~T}_{1} \mathrm{P}_{1}$ had significantly the highest $(639.17 \mathrm{mg} / 100 \mathrm{~g})$ vitamin $\mathrm{C}$ content of juice. The increase in storage period, the vitamin $\mathrm{C}$ content of juice was decreased in all the treatment combinations, but it was less in $\mathrm{P}_{1}$ and $\mathrm{F}_{1}$ treatment combination. This could be due to fruit was fresh at time $T_{1}$ and packed in $\mathrm{P}_{1}$ maintained higher vitamin $\mathrm{C}$ content during storage period.

\section{Taste of candy (Prepared from 4 and 8 month after storage of fruits)}

The individual effect of F, C and P (Table 8) on taste of candy prepared from 4 and 8 months of storage gave significant results. Candy prepared after 4 months storage, fruit $F_{1}$, treated with chemical $C_{1}$ and packed in $P_{1}$ recorded maximum organoleptic score of taste (6.71, 6.69 and 7.10) respectively. After 8 months of storage candy prepared from packing $\mathrm{P}_{1}$ contained significantly higher (6.80) organoleptic score of taste. The fruit $F_{1}$, anti-darkening chemicals $\mathrm{C}_{1}$ and packing $\mathrm{P}_{1}$ contained significantly higher organoleptic score. This may be due to in fruit $F_{1}$ and packing $\mathrm{P}_{1}$ the rate of physico chemical changes ware less, which helps to reduce respiration and oxidation. 
Table.1 Individual effect of F, P and T on vitamin-C content of fresh and frozen aonla candy

\begin{tabular}{|c|c|c|c|c|c|}
\hline Treatments & $\mathbf{1}$ & $\mathbf{2}$ & $\mathbf{3}$ & C.D at 5\% & S. Em. \pm \\
\hline $\mathbf{F}$ & 170.02 & 163.15 & & $\mathbf{1 . 0 5}$ & $\mathbf{0 . 3 8}$ \\
\hline $\mathbf{P}$ & 170.00 & 164.39 & 165.36 & $\mathbf{1 . 2 9}$ & $\mathbf{0 . 4 6}$ \\
\hline $\mathbf{T}$ & 203.07 & 162.57 & 134.12 & $\mathbf{1 . 2 9}$ & $\mathbf{0 . 4 6}$ \\
\hline
\end{tabular}

Table.2 Interaction effect of $\mathrm{F} \times \mathrm{P}$ and $\mathrm{F} \times \mathrm{T}$ on vitamin-C content of fresh and frozen

\begin{tabular}{|c|c|c|c|c|c|c|}
\hline Treatments & $\mathbf{P}_{\mathbf{1}}$ & $\mathbf{P}_{\mathbf{2}}$ & $\mathbf{P}_{\mathbf{3}}$ & $\mathbf{T}_{\mathbf{1}}$ & $\mathbf{T}_{\mathbf{2}}$ & $\mathbf{T}_{\mathbf{3}}$ \\
\hline $\mathbf{F}_{\mathbf{1}}$ & 174.59 & 167.30 & 168.15 & 203.26 & 167.14 & 139.65 \\
\hline $\mathbf{F}_{\mathbf{2}}$ & 165.41 & 161.48 & 162.57 & 202.87 & 157.99 & 128.59 \\
\hline C.D at 5\% & & $\mathbf{1 . 8 2}$ & & & $\mathbf{1 . 8 2}$ & \\
\hline S. Em. $\mathbf{\pm}$ & & $\mathbf{0 . 6 5}$ & & & $\mathbf{0 . 6 5}$ & \\
\hline
\end{tabular}

\begin{tabular}{|c|c|c|c|}
\hline \multicolumn{4}{|c|}{ Table.3 Interaction effect of P×T on vitamin-C content of fresh and frozen } \\
\hline aonla candy & \multicolumn{3}{|c|}{} \\
\hline Treatments & $\mathbf{P}_{\mathbf{1}}$ & $\mathbf{P}_{\mathbf{2}}$ & $\mathbf{P}_{\mathbf{3}}$ \\
\hline $\mathbf{T}_{\mathbf{1}}$ & 203.30 & 202.04 & 203.86 \\
\hline $\mathbf{T}_{\mathbf{2}}$ & 168.34 & 159.74 & 159.62 \\
\hline $\mathbf{T}_{\mathbf{2}}$ & 138.37 & 131.39 & 132.60 \\
\hline C.D at 5\% & & $\mathbf{2 . 2 3}$ & \\
\hline S. Em. \pm & & $\mathbf{0 . 8 0}$ & \\
\hline
\end{tabular}

\begin{tabular}{|c|c|c|c|}
\hline \multicolumn{4}{|c|}{ Table.4 Interaction effect of $\mathrm{F} \times \mathrm{C} \times \mathrm{T}$ on vitamin-C content of fresh and } \\
frozen aonla candy \\
\hline Treatments & $\mathbf{T}_{\mathbf{1}}$ & $\mathbf{T}_{\mathbf{2}}$ & $\mathbf{T}_{\mathbf{3}}$ \\
\hline $\mathbf{F}_{\mathbf{1}} \mathbf{C}_{\mathbf{1}}$ & 203.41 & 167.08 & 140.78 \\
\hline $\mathbf{F}_{\mathbf{1}} \mathbf{C}_{\mathbf{2}}$ & 202.54 & 168.05 & 139.73 \\
\hline $\mathbf{F}_{\mathbf{1}} \mathbf{C}_{\mathbf{3}}$ & 203.83 & 166.30 & 138.43 \\
\hline $\mathbf{F}_{\mathbf{2}} \mathbf{C}_{\mathbf{1}}$ & 202.34 & 159.88 & 127.81 \\
\hline $\mathbf{F}_{\mathbf{2}} \mathbf{C}_{\mathbf{2}}$ & 203.04 & 158.43 & 126.94 \\
\hline $\mathbf{F}_{\mathbf{2}} \mathbf{C}_{\mathbf{3}}$ & 203.24 & 155.67 & 131.03 \\
\hline C.D $\mathbf{a t} \mathbf{5 \%}$ & & $\mathbf{3 . 1 6}$ & \\
\hline S. $\mathbf{E}$. $\mathbf{\pm}$ & & $\mathbf{1 . 1 3}$ & \\
\hline
\end{tabular}

Table.5 Individual effect of F, P and T on vitamin-C content of fresh and frozen aonla juice

\begin{tabular}{|c|c|c|c|c|c|c|}
\hline Treatments & $\mathbf{1}$ & $\mathbf{2}$ & $\mathbf{3}$ & C.D at 5\% & S. Em. \pm & C.V \% \\
\hline $\mathbf{F}$ & 546.58 & 503.04 & & $\mathbf{3 . 3 5}$ & $\mathbf{1 . 2 0}$ & $\mathbf{2 . 0 5}$ \\
\hline $\mathbf{P}$ & 539.30 & 520.55 & 514.59 & $\mathbf{4 . 1 1}$ & $\mathbf{1 . 4 7}$ & \\
\hline $\mathbf{T}$ & 603.91 & 507.29 & 463.22 & $\mathbf{4 . 1 1}$ & $\mathbf{1 . 4 7}$ & \\
\hline
\end{tabular}


Table.6 Interaction effect of $\mathrm{F} \times \mathrm{P}$ and $\mathrm{P} \times \mathrm{T}$ on vitamin-C content of fresh and frozen aonla juice

\begin{tabular}{|c|c|c|c|c|c|}
\hline Treatments & $\mathbf{F}_{1}$ & $\mathbf{F}_{2}$ & $\mathbf{T}_{1}$ & $\mathbf{T}_{2}$ & $\mathbf{T}_{3}$ \\
\hline $\mathbf{P}_{1}$ & 558.38 & 520.21 & 618.38 & 526.95 & 472.56 \\
\hline $\mathbf{P}_{2}$ & 545.61 & 495.49 & 600.85 & 500.52 & 460.27 \\
\hline $\mathbf{P}_{3}$ & 535.74 & 493.43 & 592.51 & 494.41 & 456.84 \\
\hline C.D at $5 \%$ & \multicolumn{2}{|c|}{5.81} & \multicolumn{3}{|c|}{7.12} \\
\hline S. Em. \pm & \multicolumn{2}{|c|}{2.07} & \multicolumn{3}{|c|}{2.54} \\
\hline
\end{tabular}

Table.7 Interaction effect of $\mathrm{F} \times \mathrm{C} \times \mathrm{T}$ and $\mathrm{F} \times \mathrm{P} \times \mathrm{T}$ on vitamin- $\mathrm{C}$ content of fresh and frozen aonla juice

\begin{tabular}{|c|c|c|c|c|c|c|}
\hline Treatments & $\mathbf{C}_{\mathbf{1}}$ & $\mathbf{C}_{\mathbf{2}}$ & $\mathbf{C}_{\mathbf{3}}$ & $\mathbf{P}_{\mathbf{1}}$ & $\mathbf{P}_{\mathbf{2}}$ & $\mathbf{P}_{\mathbf{3}}$ \\
\hline $\mathbf{F}_{\mathbf{1}} \mathbf{T}_{\mathbf{1}}$ & 629.56 & 627.06 & 620.06 & 639.17 & 624.78 & 612.74 \\
\hline $\mathbf{F}_{\mathbf{1}} \mathbf{T}_{\mathbf{2}}$ & 530.30 & 532.18 & 522.18 & 540.24 & 525.95 & 525.95 \\
\hline $\mathbf{F}_{\mathbf{1}} \mathbf{T}_{\mathbf{3}}$ & 477.95 & 485.56 & 493.71 & 495.73 & 486.09 & 486.09 \\
\hline $\mathbf{F}_{\mathbf{2}} \mathbf{T}_{\mathbf{1}}$ & 580.29 & 577.36 & 589.14 & 597.59 & 576.93 & 572.27 \\
\hline $\mathbf{F}_{\mathbf{2}} \mathbf{T}_{\mathbf{2}}$ & 482.74 & 488.38 & 487.36 & 513.67 & 475.09 & 469.73 \\
\hline $\mathbf{F}_{\mathbf{2}} \mathbf{T}_{\mathbf{3}}$ & 443.64 & 436.40 & 442.09 & 449.39 & 434.45 & 438.29 \\
\hline C.D at 5\% & & $\mathbf{1 0 . 0 6}$ & & & $\mathbf{1 0 . 0 6}$ & \\
\hline S. $\mathbf{E m} \mathbf{\pm}$ & & $\mathbf{3 . 5 9}$ & & & $\mathbf{3 . 5 9}$ & \\
\hline
\end{tabular}

Table.8 Individual effect of F, C and P on taste of candy prepared from 4 and 8 month of storage fruits

\begin{tabular}{|c|c|c|c|c|c|c|}
\hline & \multicolumn{3}{|c|}{ 4 month of storage } & \multicolumn{3}{c|}{ 8 month of storage } \\
\hline Treatments & $\mathbf{1}$ & $\mathbf{2}$ & $\mathbf{3}$ & $\mathbf{1}$ & $\mathbf{2}$ & $\mathbf{3}$ \\
\hline F & 6.71 & 6.25 & & - & - & - \\
\hline C & 6.69 & 6.31 & 6.44 & - & - & - \\
\hline P & 7.10 & 5.74 & 6.60 & 6.80 & 6.01 & 5.89 \\
\hline C.D at 5\% & & $\mathbf{0 . 1 9}$ & & & $\mathbf{0 . 1 6}$ & \\
\hline S. Em. \pm & & $\mathbf{0 . 0 7}$ & & & $\mathbf{0 . 0 5}$ \\
\hline
\end{tabular}

Table.9 Interaction effects of $\mathrm{F} \times \mathrm{C}$ on taste of candy prepared from 4 and 8 month of storage fruits

\begin{tabular}{|c|c|c|c|c|c|c|}
\hline & \multicolumn{3}{|c|}{ month of storage } & \multicolumn{3}{c|}{ 8 month of storage } \\
\hline Treatments & $\mathbf{C}_{\mathbf{1}}$ & $\mathbf{C}_{\mathbf{2}}$ & $\mathbf{C}_{\mathbf{3}}$ & $\mathbf{C}_{\mathbf{1}}$ & $\mathbf{C}_{\mathbf{2}}$ & $\mathbf{C}_{\mathbf{3}}$ \\
\hline $\mathbf{F}_{\mathbf{1}}$ & 7.09 & 6.40 & 6.63 & 6.42 & 6.30 & 6.01 \\
\hline $\mathbf{F}_{\mathbf{2}}$ & 6.29 & 6.21 & 6.25 & 6.35 & 6.28 & 6.01 \\
\hline C.D at 5\% & \multicolumn{3}{|c|}{$\mathbf{0 . 2 7}$} & & $\mathbf{0 . 2 2}$ & \\
\hline S. Em. $\mathbf{E}$ & & $\mathbf{0 . 0 9}$ & & $\mathbf{0 . 0 8}$ \\
\hline
\end{tabular}


Table.10 Interaction effect of $\mathrm{F} \times \mathrm{C} \times \mathrm{P}$ on taste of candy prepared from 4 and 8 month of storage fruits

\begin{tabular}{|c|c|c|c|c|c|c|}
\hline & \multicolumn{3}{|c|}{ 4 month of storage } & \multicolumn{3}{|c|}{$\mathbf{8}$ month of storage } \\
\hline Treatments & $\mathbf{P}_{\mathbf{1}}$ & $\mathbf{P}_{\mathbf{2}}$ & $\mathbf{P}_{\mathbf{3}}$ & $\mathbf{P}_{\mathbf{1}}$ & $\mathbf{P}_{\mathbf{2}}$ & $\mathbf{P}_{\mathbf{3}}$ \\
\hline $\mathbf{F}_{\mathbf{1}} \mathbf{C}_{\mathbf{1}}$ & 7.62 & 6.26 & 7.04 & 7.08 & 5.42 & 5.52 \\
\hline $\mathbf{F}_{\mathbf{1}} \mathbf{C}_{\mathbf{2}}$ & 7.16 & 5.80 & 6.25 & 6.31 & 6.36 & 6.25 \\
\hline $\mathbf{F}_{\mathbf{1}} \mathbf{C}_{\mathbf{3}}$ & 7.16 & 6.03 & 6.71 & 6.98 & 6.36 & 5.94 \\
\hline $\mathbf{F}_{\mathbf{2}} \mathbf{C}_{\mathbf{1}}$ & 6.94 & 5.57 & 6.37 & 6.78 & 6.36 & 5.94 \\
\hline $\mathbf{F}_{\mathbf{2}} \mathbf{C}_{\mathbf{2}}$ & 6.71 & 5.68 & 6.25 & 6.88 & 5.94 & 6.04 \\
\hline $\mathbf{F}_{\mathbf{2}} \mathbf{C}_{\mathbf{3}}$ & 7.05 & 5.11 & 6.60 & 6.78 & 5.63 & 5.63 \\
\hline $\mathbf{C . D}$ at 5\% & & $\mathbf{0 . 4 6}$ & & & $\mathbf{0 . 3 8}$ & \\
\hline $\mathbf{S . ~} \mathbf{E m} \mathbf{\pm}$ & & $\mathbf{0 . 1 6}$ & & & $\mathbf{0 . 1 3}$ & \\
\hline
\end{tabular}

Table.11 Individual effect of packing materials on marketability of candy prepared from 4 and 8 month of storage fruits

\begin{tabular}{|c|c|c|c|c|c|c|}
\hline & \multicolumn{2}{|c|}{$\mathbf{4}$ month of storage } & \multicolumn{3}{|c|}{$\mathbf{8}$ month of storage } \\
\hline Treatments & $\mathbf{1}$ & $\mathbf{2}$ & $\mathbf{3}$ & $\mathbf{1}$ & $\mathbf{2}$ & $\mathbf{3}$ \\
\hline $\mathbf{P}$ & 6.98 & 5.86 & 6.39 & 6.70 & 6.09 & 5.77 \\
\hline C & - & - & - & 6.32 & 6.20 & 6.04 \\
\hline C.D at 5\% & & $\mathbf{0 . 3 0}$ & & & $\mathbf{0 . 1 8}$ & \\
\hline S. Em. \pm & & $\mathbf{0 . 1 0}$ & & & $\mathbf{0 . 0 6}$ & \\
\hline
\end{tabular}

Table.12 Interaction effects of $\mathrm{F} \times \mathrm{P}$ on marketability of candy prepared from 4 and 8 month of storage fruits

\begin{tabular}{|c|c|c|c|c|c|c|}
\hline & \multicolumn{3}{|c|}{ 4 month of storage } & \multicolumn{3}{c|}{ 8 month of storage } \\
\hline Treatments & $\mathbf{P}_{\mathbf{1}}$ & $\mathbf{P}_{\mathbf{2}}$ & $\mathbf{P}_{\mathbf{3}}$ & $\mathbf{P}_{\mathbf{1}}$ & $\mathbf{P}_{\mathbf{2}}$ & $\mathbf{P}_{\mathbf{3}}$ \\
\hline $\mathbf{F}_{\mathbf{1}}$ & 7.01 & 6.21 & 6.36 & 6.70 & 6.32 & 5.63 \\
\hline $\mathbf{F}_{\mathbf{2}}$ & 6.95 & 5.51 & 6.42 & 6.07 & 5.87 & 5.90 \\
\hline C.D at 5\% & & $\mathbf{0 . 4 2}$ & & & $\mathbf{0 . 2 5}$ & \\
\hline S. Em. $\mathbf{E}$ & & $\mathbf{0 . 1 5}$ & & & $\mathbf{0 . 0 9}$ \\
\hline
\end{tabular}

Table.13 Interaction effect of $\mathrm{F} \times \mathrm{C} \times \mathrm{P}$ on marketability of candy prepared from 4 and 8 month of storage fruits

\begin{tabular}{|c|c|c|c|c|c|c|}
\hline & \multicolumn{3}{|c|}{ 4 month of storage } & \multicolumn{3}{c|}{ 8 month of storage } \\
\hline Treatments & $\mathbf{P}_{\mathbf{1}}$ & $\mathbf{P}_{\mathbf{2}}$ & $\mathbf{P}_{\mathbf{3}}$ & $\mathbf{P}_{\mathbf{1}}$ & $\mathbf{P}_{\mathbf{2}}$ & $\mathbf{P}_{\mathbf{3}}$ \\
\hline $\mathbf{F}_{\mathbf{1}} \mathbf{C}_{\mathbf{1}}$ & 7.50 & 6.71 & 6.36 & 7.19 & 5.84 & 5.52 \\
\hline $\mathbf{F}_{\mathbf{1}} \mathbf{C}_{\mathbf{2}}$ & 6.94 & 6.03 & 6.60 & 6.98 & 5.94 & 6.15 \\
\hline $\mathbf{F}_{\mathbf{1}} \mathbf{C}_{\mathbf{3}}$ & 6.59 & 5.91 & 6.14 & 6.98 & 6.15 & 5.21 \\
\hline $\mathbf{F}_{\mathbf{2}} \mathbf{C}_{\mathbf{1}}$ & 6.60 & 5.43 & 6.89 & 6.88 & 6.04 & 6.73 \\
\hline $\mathbf{F}_{\mathbf{2}} \mathbf{C}_{\mathbf{2}}$ & 6.98 & 5.57 & 6.02 & 6.77 & 5.94 & 6.15 \\
\hline $\mathbf{F}_{\mathbf{2}} \mathbf{C}_{\mathbf{3}}$ & 7.28 & 5.52 & 6.37 & 6.46 & 5.63 & 5.88 \\
\hline C.D at 5\% & & $\mathbf{0 . 7 2}$ & & & $\mathbf{0 . 4 4}$ & \\
\hline S. $\mathbf{E}$. $\mathbf{\pm}$ & & $\mathbf{0 . 2 5}$ & & & $\mathbf{0 . 1 5}$ & \\
\hline
\end{tabular}




\begin{tabular}{|c|c|c|c|c|c|c|}
\hline \multicolumn{8}{|c|}{ Table.14 Individual effect of F. C, P on taste of juice prepared from 4 and 8} \\
month of storage fruits \\
\hline Treatments & $\mathbf{4}$ & $\mathbf{4}$ month of storage & \multicolumn{3}{c|}{$\mathbf{8}$ month of storage } \\
\hline F & - & - & - & 6.75 & 5.75 & \\
\hline C & 7.22 & 6.39 & 6.60 & 6.54 & 6.16 & 6.04 \\
\hline P & 7.22 & 6.46 & 6.53 & 6.75 & 6.21 & 5.79 \\
\hline C.D at 5\% & & $\mathbf{0 . 3 2}$ & & & $\mathbf{0 . 2 5}$ & \\
\hline S. Em. \pm & & $\mathbf{0 . 1 1}$ & & & $\mathbf{0 . 0 9}$ & \\
\hline
\end{tabular}

Table.15 Interaction effect of $\mathrm{F} \times \mathrm{C} \times \mathrm{P}$ on taste of juice prepared from 4 and 8 month of storage fruits

\begin{tabular}{|c|c|c|c|c|c|c|}
\hline & \multicolumn{3}{|c|}{ 4 month of storage } & \multicolumn{3}{c|}{ 8 month of storage } \\
\hline Treatments & $\mathbf{P}_{\mathbf{1}}$ & $\mathbf{P}_{\mathbf{2}}$ & $\mathbf{P}_{\mathbf{3}}$ & $\mathbf{P}_{\mathbf{1}}$ & $\mathbf{P}_{\mathbf{2}}$ & $\mathbf{P}_{\mathbf{3}}$ \\
\hline $\mathbf{F}_{\mathbf{1}} \mathbf{C}_{\mathbf{1}}$ & 7.92 & 7.50 & 6.67 & 7.50 & 7.00 & 6.50 \\
\hline $\mathbf{F}_{\mathbf{1}} \mathbf{C}_{\mathbf{2}}$ & 7.09 & 6.25 & 6.25 & 7.00 & 6.50 & 6.50 \\
\hline $\mathbf{F}_{\mathbf{1}} \mathbf{C}_{\mathbf{3}}$ & 7.09 & 5.83 & 6.67 & 6.75 & 6.25 & 6.00 \\
\hline $\mathbf{F}_{\mathbf{2}} \mathbf{C}_{\mathbf{1}}$ & 6.67 & 7.09 & 7.05 & 6.50 & 6.00 & 6.25 \\
\hline $\mathbf{F}_{\mathbf{2}} \mathbf{C}_{\mathbf{2}}$ & 7.09 & 6.25 & 5.83 & 6.00 & 5.75 & 5.50 \\
\hline $\mathbf{F}_{\mathbf{2}} \mathbf{C}_{\mathbf{3}}$ & 6.67 & 6.25 & 6.67 & 5.50 & 5.25 & 5.00 \\
\hline $\mathbf{C . D} \mathbf{a t} \mathbf{5 \%}$ & & $\mathbf{0 . 5 8}$ & & & $\mathbf{0 . 4 5}$ & \\
\hline $\mathbf{S} . \mathbf{E m} \mathbf{\pm}$ & & $\mathbf{0 . 2 0}$ & & & $\mathbf{0 . 1 6}$ \\
\hline
\end{tabular}

Table.16 Individual effect of F. C, P on marketability of juice prepared from 4 and 8 month of storage fruits

\begin{tabular}{|c|c|c|c|c|c|c|}
\hline & \multicolumn{3}{|c|}{$\mathbf{4}$ month of storage } & \multicolumn{3}{|c|}{ 8 month of storage } \\
\hline Treatments & $\mathbf{1}$ & $\mathbf{2}$ & $\mathbf{3}$ & $\mathbf{1}$ & $\mathbf{2}$ & $\mathbf{3}$ \\
\hline F & - & - & - & 6.65 & 6.25 & \\
\hline P & 7.35 & 6.47 & 6.32 & 6.88 & 6.39 & 6.08 \\
\hline C.D at 5\% & & $\mathbf{0 . 4 8}$ & & & $\mathbf{0 . 2 9}$ & \\
\hline S. Em. \pm & & $\mathbf{0 . 1 7}$ & & & $\mathbf{0 . 0 9}$ \\
\hline
\end{tabular}

Table.17 Interaction effect of $\mathrm{F} \times \mathrm{C} \times \mathrm{P}$ on marketability of juice prepared from 4 and 8 month of storage fruits

\begin{tabular}{|c|c|c|c|c|c|c|}
\hline & \multicolumn{3}{|c|}{ 4 month of storage } & \multicolumn{3}{c|}{ 8 month of storage } \\
\hline Treatments & $\mathbf{P}_{\mathbf{1}}$ & $\mathbf{P}_{\mathbf{2}}$ & $\mathbf{P}_{\mathbf{3}}$ & $\mathbf{P}_{\mathbf{1}}$ & $\mathbf{P}_{\mathbf{2}}$ & $\mathbf{P}_{\mathbf{3}}$ \\
\hline $\mathbf{F}_{\mathbf{1}} \mathbf{C}_{\mathbf{1}}$ & 7.92 & 7.67 & 7.09 & 7.25 & 7.00 & 7.00 \\
\hline $\mathbf{F}_{\mathbf{1}} \mathbf{C}_{\mathbf{2}}$ & 7.09 & 6.75 & 6.25 & 6.58 & 6.75 & 6.00 \\
\hline $\mathbf{F}_{\mathbf{1}} \mathbf{C}_{\mathbf{3}}$ & 6.67 & 6.67 & 6.25 & 6.75 & 6.50 & 6.00 \\
\hline $\mathbf{F}_{\mathbf{2}} \mathbf{C}_{\mathbf{1}}$ & 6.84 & 7.09 & 7.05 & 6.75 & 6.50 & 6.75 \\
\hline $\mathbf{F}_{\mathbf{2}} \mathbf{C}_{\mathbf{2}}$ & 6.25 & 5.84 & 6.67 & 6.00 & 6.50 & 6.50 \\
\hline $\mathbf{F}_{\mathbf{2}} \mathbf{C}_{\mathbf{3}}$ & 6.25 & 6.25 & 5.84 & 6.00 & 5.50 & 5.75 \\
\hline C.D at 5\% & & $\mathbf{0 . 8 6}$ & & & $\mathbf{0 . 5 2}$ & \\
\hline S. Em. $\mathbf{E}$ & & $\mathbf{0 . 3 0}$ & & & $\mathbf{0 . 1 8}$ \\
\hline
\end{tabular}




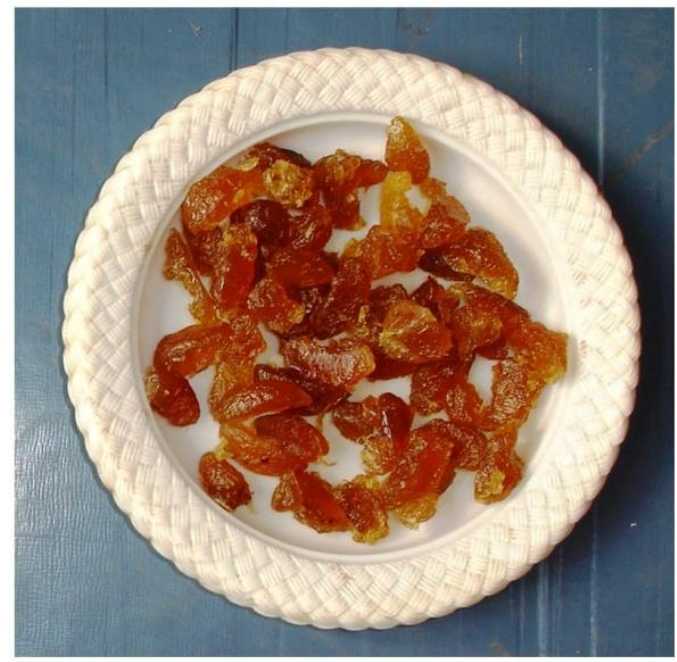

$$
\begin{array}{llll}
\mathrm{F}_{1} & \mathrm{C}_{1} & \mathrm{P}_{1}
\end{array}
$$

\section{After 4 month}

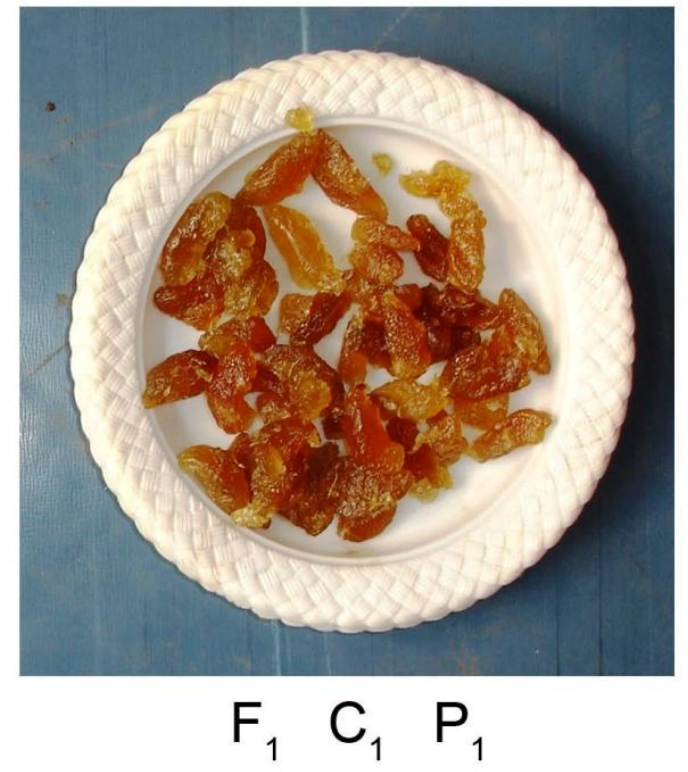

After 8 month

\section{Plate 1 : Comparision of candy}

$\mathrm{C}_{1}$ had anti darkening properties, so it reduced the phenolic activity which helps to controlled fruit darkening during storage.

Therefore, those fruits maintained their natural taste during candy preparation and recorded higher organoleptic score.
The $\mathrm{F} \times \mathrm{C}$ (Table 9) and $\mathrm{F} \times \mathrm{C} \times \mathrm{P}$ (Table 10) interactions exhibits significant variation for taste of candy prepared from 4 and 8 months of frozen aonla fruits. After 4 and 8 months of storage the treatment combination of $\mathrm{F}_{1} \mathrm{C}_{1}$ recorded significantly higher (7.09 and 6.42) organoleptic score of candy taste which was 
at par with $\mathrm{F}_{1} \mathrm{C}_{2}, \mathrm{~F}_{2} \mathrm{C}_{1}$ and $\mathrm{F}_{2} \mathrm{C}_{2}$ for 8 months of storage. In $\mathrm{F} \times \mathrm{C} \times \mathrm{P}$ interaction, significantly the highest organoleptic score of candy taste was recorded after 4 and 8 months of storage in treatment combination of $\mathrm{F}_{1} \mathrm{C}_{1} \mathrm{P}_{1}$ (7.62 and 7.08) respectively, which was being at par with $\mathrm{F}_{1} \mathrm{C}_{2} \mathrm{P}_{1}$ and $\mathrm{F}_{1} \mathrm{C}_{3} \mathrm{P}_{1}$ after 4 months of storage and $\mathrm{F}_{1} \mathrm{C}_{3} \mathrm{P}_{1}, \quad \mathrm{~F}_{2} \mathrm{C}_{1} \mathrm{P}_{1}, \quad \mathrm{~F}_{2} \mathrm{C}_{2} \mathrm{P}_{1}$ and $\mathrm{F}_{2} \mathrm{C}_{3} \mathrm{P}_{1}$ after 8 months of storage. This might be due to the combined effect of $F_{1}$ fruit and $\mathrm{P}_{1}$ packing and chemical $\mathrm{C}_{1}$ maintained natural taste during storage because of slower changes in physico chemical properties and hence recorded more organoleptic score of candy.

\section{Marketability of candy (Prepared from 4 and 8 months after storage of fruits)}

The individual effect of $\mathrm{P}$ and $\mathrm{C}, \mathrm{P}$ (Table 11) on marketability of candy prepared from 4 month and 8 months of storage fruits gave significant result respectively. Fruits packed in $\mathrm{P}_{1}$ recorded the highest (6.98 and 6.70) organoleptic score of candy marketability after 4 and 8 months of storage respectively. This may be due to the reason that packing $\mathrm{P}_{1}$ kept their natural physical and chemical characters by retarding oxidation and respiration, so candy prepared from those fruits had good marketability. Chemicals $C_{1}$ contained significantly higher (6.32) organoleptic score for marketability, which was at par with $\mathrm{C}_{2}$ after 8 months of storage.

The $\mathrm{F} \times \mathrm{P}($ Table 12) and $\mathrm{F} \times \mathrm{C} \times \mathrm{P}$ (Table 13) interactions recorded significant for marketability of candy prepared from 4 and 8 months after storage of fruits. The treatment combination of $\mathrm{F}_{1} \mathrm{P}_{1}$ registered significantly the highest (7.01 and 6.70) organoleptic score of candy marketability prepared after 4 and 8 months of storage which was at par with $\mathrm{F}_{2} \mathrm{P}_{1}$ for 4 months after storage. In $\mathrm{F} \times \mathrm{C} \times \mathrm{P}$ interaction maximum $(7.50$ and 7.19$)$ organoleptic score for marketability was observed in treatment combination of $\mathrm{F}_{1} \mathrm{C}_{1} \mathrm{P}_{1}$ after 4 and 8 months of storage respectively, compared to all other treatment combinations except $\mathrm{F}_{1} \mathrm{C}_{2} \mathrm{P}_{1}, \mathrm{~F}_{2} \mathrm{C}_{2} \mathrm{P}_{1}$ and $\mathrm{F}_{2} \mathrm{C}_{3} \mathrm{P}_{1}$ after 4 months of storage and $\mathrm{F}_{1} \mathrm{C}_{2} \mathrm{P}_{1}, \mathrm{~F}_{1} \mathrm{C}_{3} \mathrm{P}_{1}, \mathrm{~F}_{2} \mathrm{C}_{1} \mathrm{P}_{1}$ and $\mathrm{F}_{2} \mathrm{C}_{2} \mathrm{P}_{1}$ after 8 months of storage. In both the interactions fruit $\mathrm{F}_{1}$, Treated with chemical $\mathrm{C}_{1}$ and packed in $\mathrm{P}_{1}$ registered good marketability. This may be due to the fact that in $\mathrm{F}_{1}, \mathrm{C}_{1}$ and $\mathrm{P}_{1}$ fruits maintained their natural physical and chemical properties during storage because of less oxidation and respiration.

\section{Taste of juice (Prepared from 4 and 8 month of storage fruits)}

The individual effect of $\mathrm{C}, \mathrm{P}$ and $\mathrm{F}, \mathrm{C}, \mathrm{P}$ were found significant for taste of juice prepared from 4 and 8 month of storage fruits respectively (Table 14). Chemical $\mathrm{C}_{1}$ and packing $\mathrm{P}_{1}$ recorded maximum (7.22 and 7.22) organoleptic score of taste after 4 months of storage respectively. Juice prepared from fruit $\mathrm{F}_{1}$, treated with chemical $\mathrm{C}_{1}$ and packed in $\mathrm{P}_{1}$ recorded maximum $(6.75,6.54$ and 6.75) organoleptic score of taste after 8 months of storage respectively. This may be due to the reason that in fruit $F_{1}$ and packing $\mathrm{P}_{1}$, the rate of physico chemical changes was decrease because of less respiration and oxidation while chemical $\mathrm{C}_{1}$ had anti darkening property which controlled the phenolic activity and thereby controlled the fruit darkening during storage. Hence, those fruits maintained their natural taste and therefore, juice prepared from those fruits registered higher organoleptic score.

The interaction effect between taste of fresh fruit juice and juice prepared from four and eight month of storage fruit was significant (Table 15). The highest (7.92 and 7.50) organoleptic score was obtained in the treatment combination of $\mathrm{F}_{1} \mathrm{C}_{1} \mathrm{P}_{1}$ after four and eight month of storage which was at par 
with fresh fruits and $\mathrm{F}_{1} \mathrm{C}_{2} \mathrm{P}_{1}$ after 4 months of storage. This may be due to the reason that combined effect of fruit $F_{1}$, chemical $C_{1}$ and $\mathrm{C}_{2}$ and $\mathrm{P}_{1}$ packing.

\section{Marketability of juice (prepared from 4 and 8 month of storage fruits)}

The individual effect of $\mathrm{P}$ and $\mathrm{F}, \mathrm{P}$ on marketability of juice prepared from 4 and 8 month of storage was significant respectively (Table 16). Maximum (7.35) organoleptic score of juice marketability was noted in the treatment $\mathrm{P}_{1}$ after 4 months of storage and Fruit $\mathrm{F}_{1}$ and packing $\mathrm{P}_{1}$ contained significantly the highest (6.65 and 6.88) organoleptic score of juice marketability after 8 months of storage respectively. The interaction effect between marketability of fresh fruit juice and juice prepared from four month of storage fruits was significant. The highest (7.92 and 7.25) organoleptic score was registered with $\mathrm{F}_{1} \mathrm{C}_{1} \mathrm{P}_{1}$ after 4 and 8 months of storage respectively (Table 17). It was at par with $\mathrm{F}_{1} \mathrm{C}_{2} \mathrm{P}_{1}$ after 4 and 8 months of storage. This may be possible because of fresh fruit contained its natural quality as well as treatment combinations of $\mathrm{F}_{1} \mathrm{C}_{1} \mathrm{P}_{1}$ and $\mathrm{F}_{1} \mathrm{C}_{2} \mathrm{P}_{1}$ also maintained natural quality of fruits even after 4 month of storage because of slow changes in physico chemical property and therefore juice prepared from those fruits recorded more score of marketability compared to other treatments.

\section{References}

Nath, V., Singh, I. S., Sanjeev Kumar and Pandey, A. K. (1992). Effect of postharvest treatments on shelf life of aonla fruits. Prog. Hort., 24 (1-2): 79-82.

Patra, D. K and Sadhu, M. K. (1992). Influence of post-harvest calcium treatment on shelf life and quality of litchi fruits. South Indian Horticulture, 40 (5): 252-256.

Ranganna, S. (1979). "Manual of analysis of fruits and vegetable products". Tata Mc Graw Hill Publishing Co. Ltd., New Delhi.

Ray, N. R. and Kikani, K. P. (2003). Value addition in amla (Candy processing). Amla in India: Aonla Growers Association, Salem @ 2005. pp. 212214.

Singh, I. S. and Sanjeev Kumar (1995). Studies on processing of Amla fruits II. Amla products, Prog. Hort. 27 (1-2): 39-47.

Singh, I. S., Pathak, R. K., Diwedi, R. and Singh, H. K. (1993). Post- harvest handling in Amla production and postharvest technology. NDUAT, Faizabad. pp. 19-24.

Thakur, B. R. and Arya, S. S. (1988). Relative suitability of plastic films for the frozen storage of mango pulp. J. Food Processing and preservation. 12(3): 171-178 Mapson, 1970

\section{How to cite this article:}

Patel, V.K., B.H. Panchal and Khimani, R.A. 2018. Effect of Anti Darkening Treatments and Packing Materials on Whole and Sliced Fruits of Frozen Aonla Processed Product cv. Gujarat Aonla-1. Int.J.Curr.Microbiol.App.Sci. 7(11): 592-601. doi: https://doi.org/10.20546/ijcmas.2018.711.072 